Ann. Zootech., rg69, 18 (2), 22I-226.

\title{
MÉTHODE D'OBSERVATION DE L'EMPLOI DU TEMPS DES TROUPEAUX DE VACHES ALLAITANTES AU PATURAGE
}

\author{
M. PETIT \\ Station de Recherches sur l'Élevage des Ruminants, \\ Centre de Recherches zootechniques et vétérinaires sur les Ruminants, \\ 63 - Saint-Genès-Champanelle \\ Institut national de la Recherche agronomique
}

\section{SOMMAIRE}

Pour mettre au point une méthode d'observation de l'emploi du temps des troupeaux de vaches allaitantes au pâturage, nous avons observé 4 vaches et 4 veaux à intervalle de 2 minutes ( 2 jours) et 6 troupeaux de 29 à 82 vaches allaitantes à intervalle de Io minutes ( 2 I jours plus 5 observations nocturnes). Un intervalle de ro minutes a été satisfaisant pour l'ensemble de 4 animaux. Pour les grands troupeaux, le pâturage peut être observé à intervalle de 20 minutes pour les veaux et de 30 minutes pour les vaches; les tétées doivent l'être à intervalle de 5 minutes. Pour une période déterminée, on peut observer les animaux pendant une seule journée quand les conditions météorologiques sont stables, et seulement de l'aube au crépuscule lorsque les jours sont longs.

\section{INTRODUCTION}

Le comportement des troupeaux de vaches allaitantes pâturant librement avec leurs veaux est très mal connu; il n'a été étudié qu'avec des troupeaux d'effectifs réduits (JOHNSTON-WALLACE et KENNEDY, I944), ou sur des pâturages très extensifs (WAGNON, I963). Nous 1'avons étudié en I965 sur 6 troupeaux exploitant des pâturages d'altitude ou "montagnes " de 1'Aubrac. Nous avons notamment cherché à préciser le temps de pâturage des vaches et des veaux ainsi que la fréquence et la durée des tétées. Les résultats enregistrés au cours de 2 I jours d'observation feront l'objet d'une prochaine publication.

Nous avons observé les animaux de façon discontinue, à intervalle de ro minutes qui était l'intervalle le plus petit possible compte tenu de la taille des troupeaux (29 à 82 vaches mères), de la surface des pâturages ( 43 à 90 ha) et du nombre réduit des observateurs ( $I$ à 2 ). Selon les auteurs, l'enregistrement des activités majeures

Annales de Zootechnie. - I969. 
telles que le pâturage peut être réalisé à intervalles allant de 4 minutes (HuGUES et REID, I95I) à 30 minutes (HULI; LOFGREEN et MEYER, I960). L'analyse de nos données complétée par certaines observations annexes nous a permis de dégager une méthode générale pour l'observation du pâturage et des tétées de troupeaux de vaches allaitantes au pâturage, méthode qui fait l'objet de la présente note. Il s'agissait de définir la fréquence des observations, leur durée journalière et le nombre de jours d'observation qui étaient nécessaires pour décrire le comportement du troupeau au cours d'une période donnée de la saison de pâturage.

\section{MATÉRIEL ET MÉTHODES}

Nous avons observé les 6 troupeaux, du lever des premières vaches le matin au coucher des dernières le soir (de 45 minutes avant le lever jusqu'à 45 minutes après le coucher du soleil environ), à intervalles de ro minutes pour le pâturage, de 5 minutes pour les tétées. Nous n'avons pas observé la rumination. A chaque enregistrement, l'observateur parcourait des yeux le troupeau, toujours dans le même sens et comptait le nombre d'animaux exerçant les différentes activités. Il le faisait rapidement, de façon à ne pas avoir à hésiter sur l'activité instantanée de certains individus; l'utilisation de jumelles a facilité ces observations.

Nous avons calculé un emploi du temps moyen en supposant constante l'activité globale du troupeau entre 2 observations successives. Si ai est le nombre d'animaux exerçant une certaine activité dans l'intervalle de $n$ minutes d'ordre $i$ compris entre le début et la fin des observations, le temps en minutes passé à cette activité par l' "animal moyen " du troupeau de A têtes, sera dans cette période supposé égal à : $\frac{\sum(a i \times n)}{\mathrm{A}}$

\section{RÉSULTATS}

\section{Fréquence des observations}

a) Pouvons-nous allonger l'intervalle entre 2 observations successives à 20 ou 30 minutes pour le pâturage, à Io ou I 5 minutes pour les tétées ?

Nous avons pris comme référence les résultats obtenus lors des 2 I jours d'observations à intervalles de Io minutes ( 5 pour les tétées), et avons calculé les écarts relatifs obtenus en prenant I mesure sur 2 ou I sur 3 à partir de la première observation (tabl. I).

Les écarts relatifs (ei) sont indifféremment positifs ou négatifs et varient indépendamment des "montagnes ", des conditions météorologiques et de la saison ; en observant à intervalles de 20 minutes, il est possible d'obtenir une erreur de moins de $5 \mathrm{p}$. Ioo dans $95 \mathrm{p}$. Ioo des cas pour le temps de pâturage des vaches et dans $90 \mathrm{p}$. Ioo des cas pour celui des veaux. On peut observer le pâturage des vaches à intervalles de 30 minutes en faisant une erreur de moins de $5 \mathrm{p}$. Ioo dans $90 \mathrm{p}$. Ioo des cas ; des résultats analogues ont été obtenus pour les temps d'ingestion des vaches en stabulation libre (Bouissou et Signoret, I965).

Les tétées au contraire doivent être observées à intervalles de 5 minutes d'autant que certaines durent moins de Io minutes (tabl. I).

b) Le problème se posait de savoir si les intervalles que nous avions initialement 
choisis de ro minutes pour le pâturage, de 5 minutes pour les tétées, suffisaient à nous fournir une estimation correcte de l'emploi du temps des animaux.

\section{TABLEAU I}

Influence de la duvée de l'intervalle entre observations

(Observation d'un troupeau à intervalles de Io, 20 ou 30 minutes)

\begin{tabular}{|c|c|c|c|c|c|c|c|c|c|}
\hline Activité considérée & \multicolumn{3}{|c|}{ Pâturage des vaches } & \multicolumn{3}{|c|}{ Pâturage des veaux } & \multicolumn{3}{|c|}{ Tétées } \\
\hline $\begin{array}{l}\text { Durée de l'intervalle entre } \\
\text { observations (en minutes) }\end{array}$ & 10 & 20 & 30 & 10 & 20 & 30 & 5 & 10 & 15 \\
\hline $\begin{array}{l}\text { Temps moyen relatif : } \\
100+\Sigma \frac{e i}{21}{ }^{(1)} \ldots \ldots \ldots \ldots \ldots\end{array}$ & 100 & 101,00 & 100,86 & 100 & 100,58 & 99,67 & 100 & 101,95 & 100,31 \\
\hline$\Sigma \frac{|e i|}{21} \cdots \cdots \cdots \cdots$ & - & 1,53 & 2,11 & $\longrightarrow$ & 2,31 & 4,23 & - & 2,79 & 3,66 \\
\hline Écart-type de $e i \ldots \ldots \ldots \ldots \ldots$ & - & 1,89 & 2,75 & - & 2,84 & 5,59 & 一 & 3,50 & 4,79 \\
\hline Probabilité $|e i|<5$ p. $100(\cdot \mathrm{p} 100)$. & - & 98 & 90 & 一 & 90 & 60 & - & 80 & 70 \\
\hline
\end{tabular}

(1) ei exprimé en p. 100 est l'écart relatif (valeur algébrique) des résultats obtenus en augmentant l'intervalle entre observations, par rapport au résultat observé à intervalle de 10 minutes (5 minutes pour les tétées).

Nous avous donc observé 4 mères et 4 veaux à intervalles de 2 minutes, les 18 et 2 I juin, de 4 h 30 à 2 I h 30 (tabl. 2). Les valeurs moyennes des temps de pâturage, obtenues en tenant compte de toutes les observations (intervalles de 2 minutes) ou d'une observation sur 5 (intervalles de Io minutes), sont très comparables et diffèrent de moins de 5 p. Ioo. Des intervalles de 2 et de 6 minutes ( 1 observation sur 3 ) donnent

\section{TABLEAU 2}

Influence de la durée de l'intervalle entre les observations

(Observations de 4 vaches avec leurs veaux à intervalles de 2 et Io minutes)

\begin{tabular}{|c|c|c|c|c|c|c|c|c|c|c|c|}
\hline \multirow{3}{*}{ Date } & \multicolumn{7}{|c|}{ Pâturage } & \multicolumn{4}{|c|}{ Tétées } \\
\hline & \multicolumn{4}{|c|}{ Vaches } & \multicolumn{3}{|c|}{ Veaux } & & \multirow[b]{2}{*}{$\begin{array}{c}\text { Temps } \\
\text { (mn) }\end{array}$} & \multirow[b]{2}{*}{$\begin{array}{l}\text { Valeur } \\
\text { relative }\end{array}$} & \multirow[b]{2}{*}{$\begin{array}{l}\text { Coeffi- } \\
\text { cient de } \\
\text { variation }\end{array}$} \\
\hline & & $\begin{array}{c}\text { Temps } \\
\text { (mn) }\end{array}$ & $\begin{array}{l}\text { Valeur } \\
\text { relative }\end{array}$ & $\begin{array}{c}\text { Coeffi- } \\
\text { cient de } \\
\text { variation }\end{array}$ & $\begin{array}{c}\text { Temps } \\
\text { (mn) }\end{array}$ & $\begin{array}{c}\text { Valeur } \\
\text { relative }\end{array}$ & $\begin{array}{c}\text { Coeffi- } \\
\text { cient de } \\
\text { variation }\end{array}$ & & & & \\
\hline \multirow{2}{*}{$18-6$} & $2 \mathrm{mn}$ & 482 & 100 & 5,4 & 232 & 100 & 14,7 & $2 \mathrm{mn}$ & 40 & 100 & 22,8 \\
\hline & $10 \mathrm{mn}$ & 485 & 100,6 & 8,7 & 232 & 100 & 14,2 & $6 \mathrm{mn}$ & 42 & 105,6 & 31,0 \\
\hline \multirow{2}{*}{$21-6$} & $2 \mathrm{mn}$ & 468 & 100 & 2,9 & 225 & 100 & 16,3 & $2 \mathrm{mn}$ & 42 & 100 & 11,0 \\
\hline & $10 \mathrm{mn}$ & 460 & 98,3 & 3,5 & 235 & 104,4 & 26,1 & $6 \mathrm{mn}$ & 45 & 107,1 & 17,8 \\
\hline
\end{tabular}

pour les tétées des différences plus marquées mais inférieures à ro p. Ioo. Comme HULI et al. (I960); NELSON et FURR (I966), nous constatons que les coefficients de 
variation des temps d'activité entre individus augmentent généralement quand augmente l'intervalle entre observations, surtout pour les activités mineures comme les tétées. Cela indique que l'enregistrement du comportement individuel des animaux ou des relations entre individus (mère-veau en particulier) doit vraisemblablement être réalisé de façon continue ou à intervalle de temps très court.

Resterait à situer la valeur des observations à intervalle de 2 minutes en comparant les résultats qu'elles donnent à ceux de l'observation continue. Hugues et REID en I95I, HARKER, TAYLOR et ROLLINSON en I954, ont montré que des observations à intervalle de 4 minutes suffisent pour les activités majeures, en les comparant à 1'observation continue.

\section{Durée des observations journalières}

Les observations conduites de l'aube au crépuscule reflètent-elles bien l'activité journalière totale des animaux?

Au point du jour, les animaux se levaient et quelques veaux tétaient déjà. A la nuit tombante, toute activité, hors la rumination, cessait et les animaux se couchaient. Nous avons constaté les $14-6,8-7$ et $\mathbf{I 5}$-7, sur trois montagnes différentes, vers 0 et 2 heures, que l'activité nocturne était réduite à quelques rares tétées et à la rumination. D'une façon générale, nous retrouvions les animaux le matin aux endroits où nous les avions vus individuellement se coucher la veille. Nous pouvons donc penser que nous avons observé en juin-juillet la quasi-totalité des tétées et du pâturage journalier.

Au mois d'octobre, nous avons observé deux troupeaux différents entre 20 heures et 6 heures. Entre le coucher des derniers animaux le soir et le lever des premiers animaux le matin, 1'activité était alors relativement importante (tabl. 3).

\section{TABLEAU 3}

Activité nocturne en octobre

(p. Ioo de l'activité pour 24 heures)

\begin{tabular}{r|c|c|c|c}
\hline Date & $\begin{array}{c}\text { Conditions } \\
\text { météorologiques }\end{array}$ & \multicolumn{2}{|c|}{ Pâturage (p. 100) } & $\begin{array}{c}\text { Tétées } \\
\text { (p. 100) }\end{array}$ \\
\cline { 3 - 4 } & Vaches & Veaux & \\
\hline 11 et $7-10$ & brouillard & 2,8 & 1,8 & 23,6 \\
& nuit claire & 12,4 & 8,7 & 23,2 \\
\hline
\end{tabular}

\section{Nombre de jours d'observation}

Une journée d'observation nous fournit-elle une bonne estimation du temps d'activité journalière moyen des animaux pour une période déterminée de l'année ?

Trois séries d'observations d'un troupeau de 60 vaches allaitantes nous ont montré (tabl. 4) qu'entre journées incluses dans une période de beau temps (I8 et 2 I juin, 6 et 7 octobre), les temps de pâturage sont très comparables : la différence est inférieure à $2 \mathrm{p}$. Ioo en juin, à $4 \mathrm{p}$. Ioo en octobre. En revanche, lorsque les condi- 
tions météorologiques ne sont pas stables ( $7,8,9$ juillet), le temps de pâturage des animaux présente des variations assez fortes : jusqu'à $8 \mathrm{p}$. Ioo par rapport à la moyenne des 3 jours. Les temps de tétée sont pariois encore plus variables d'une journée à l'autre (jusqu'à $\mathrm{I} 2 \mathrm{p}$. Ioo).

TABLEAU 4

Variations journalières des temps de pâturage et de tétée

\begin{tabular}{|c|c|c|c|c|}
\hline $1^{\text {re }}$ série ( $\$$ animaux) & $\begin{array}{c}18 \text { juin } \\
\text { (beau temps) }\end{array}$ & \multicolumn{2}{|c|}{$\begin{array}{c}21 \text { juin } \\
\text { (beau temps) }\end{array}$} & $\begin{array}{c}\text { Moyenne } \\
\text { des } 2 \text { jours }\end{array}$ \\
\hline $\begin{array}{l}\text { Temps de pâturage des vaches. } \\
\text { Temps de pâturage des veaux.. } \\
\text { Temps de tétée } \ldots \ldots \ldots \ldots \ldots\end{array}$ & $\begin{array}{c}482 \mathrm{mn} \\
(101,5) \\
232 \mathrm{mn} \\
(101,5) \\
40 \mathrm{mn} \\
(97,6)\end{array}$ & \multicolumn{2}{|c|}{$\begin{array}{c}468 \mathrm{mn} \\
(98,5) \\
225 \mathrm{mn} \\
(98,5) \\
42 \mathrm{mn} \\
(402,4)\end{array}$} & $\begin{array}{c}475 \mathrm{mn} \\
(100) \\
228 \mathrm{mn} \\
(100) \\
41 \mathrm{mn} \\
(100)\end{array}$ \\
\hline $2^{\mathrm{e}}$ série (troupeau) & $\begin{array}{c}7 \text { juillet } \\
\text { (temps couvert) }\end{array}$ & $\begin{array}{c}8 \text { juillet } \\
\text { (pluie et vent) }\end{array}$ & $\begin{array}{c}9 \text { juillet } \\
\text { (temps couvert) }\end{array}$ & $\begin{array}{l}\text { Moyenne } \\
\text { des } 3 \text { jours }\end{array}$ \\
\hline $\begin{array}{l}\text { Temps de pâturage des vaches. } \\
\text { Temps de pâturage des veaux .. } \\
\text { Temps de tétée } \ldots \ldots \ldots \ldots \ldots \text {. }\end{array}$ & $\begin{array}{c}441 \mathrm{mn} \\
(96,5) \\
241 \mathrm{mn} \\
(93,4) \\
36 \mathrm{mn} \\
(83,7)\end{array}$ & $\begin{array}{c}435 \mathrm{mn} \\
(95,2) \\
259 \mathrm{mn} \\
(100,3) \\
48 \mathrm{mn} \\
(111,6)\end{array}$ & $\begin{array}{c}494 \mathrm{mn} \\
(108,2) \\
274 \mathrm{mn} \\
(106,2) \\
44 \mathrm{mn} \\
(102,3\end{array}$ & $\begin{array}{c}357 \mathrm{mn} \\
(100) \\
258 \mathrm{mn} \\
(100) \\
43 \mathrm{mn} \\
(100)\end{array}$ \\
\hline $3^{\mathrm{e}}$ série (troupeau) & $\begin{array}{c}6 \text { octobre } \\
\text { (beau temps) }\end{array}$ & \multicolumn{2}{|c|}{$\begin{array}{c}7 \text { octobre } \\
\text { (beau temps) }\end{array}$} & $\begin{array}{c}\text { Moyenne } \\
\text { des } 2 \text { jours }\end{array}$ \\
\hline $\begin{array}{l}\text { Temps de pâturage des vaches . } \\
\text { Temps de pâturage des veaux.. } \\
\text { Temps de tétée } \ldots \ldots \ldots \ldots \ldots\end{array}$ & $\begin{array}{c}518 \mathrm{mn} \\
(102,4) \\
116 \mathrm{mn} \\
(103,7) \\
32 \mathrm{mn} \\
(100)\end{array}$ & \multicolumn{2}{|c|}{$\begin{array}{l}494 \mathrm{mn} \\
(97,6) \\
386 \mathrm{mn} \\
(96,3) \\
32 \mathrm{mn} \\
(100)\end{array}$} & $\begin{array}{l}506 \mathrm{mn} \\
(100) \\
401 \mathrm{mn} \\
(100) \\
32 \mathrm{mn} \\
(100)\end{array}$ \\
\hline
\end{tabular}

Nota : Il s'agit dans les trois cas des animaux d'un même troupeau sur un même pâturage.

\section{CONCL,USIONS}

Pour des troupeaux d'effectifs importants, il est possible d'obtenir (dans 90 p. roo des cas) une erreur de moins de $5 \mathrm{p}$. Ioo en observant le pâturage des vaches toutes les 30 minutes, celui des veaux toutes les 20 minutes ; pour les tétées, il est préférable 
d'observer à intervalle de temps plus court, de 5 minutes environ. D'une façon générale, l'intervalle entre observations sera essentiellement fonction de l'activité que l'on veut enregistrer ; en effet, quand augmente la durée de l'intervalle entre observations, la variance des résultats augmente d'autant plus que l'activité considérée est plus réduite.

Pour le temps de pâturage, une journée d'observations devrait suffire, si elle est incluse dans une période de beau temps, à nous donner une bonne estimation (à moins de 5 p. Ioo près) du temps de pâturage journalier moyen au cours de cette période. Dans le cas contraire, plusieurs jours d'observations sont sans doute nécessaires.

En automne, il semble important d'observer aussi les animaux la nuit puisqu'ils peuvent pâturer d'une façon importante, tout au moins par nuit claire, et que près de $25 \mathrm{p}$. Ioo des tétées y prennent place.

Reçu pour publication en juin I969.

\section{SUMMARY}

A METHOD OF RECORDING BEHAVIOUR OF CALVES AND COWS

IN BEEF HERDS AT PASTURE

The object of this study was to establish a method of recording the suckling and grazing behaviour in herds of beef-cows at pasture. During $2 \mathrm{I}$ days (from dawn to sunset) and 5 nights, observations were make on 6 herds. Size of the herds varied between 29 and 82 cows. The animals were observed at Io $\mathrm{mn}$ intervals for grazing and at $5 \mathrm{mn}$ intervals for suckling. The recordings show that calfgrazing may be observed at $20 \mathrm{mn}$ intervals and that of cows at 3o $\mathrm{mn}$ intervals. Suckling should be recorded every 5 minutes (table I).

Four cows and their calves were studied at $2 \mathrm{mn}$ intervals for two other days, which allowed the occuracy of recording at Io $\mathrm{mn}$ intervals to be estimated (table 2). Observations must be made during the night when the days are short (table 3). A single day is sufficient for a determined period when meteorological conditions are stable (table 4 ).

\section{RÉFÉRENCES BIBLIOGRAPHIQUES}

Bouissou M.-H., Signoret J.-P., I965. Méthode d'observation du comportement des vaches en stabulation libre (résultats non publiés).

Hancock J., r953. Grazing behaviour of cattle. Anim. Breed. Abst., 21, I-I3.

HARker R. W., TAYlor J. I., Rollinson D. H. L., I954. Studies on the habits of Zebu cattle. I. Preliminary observations on grazing habits. J. Agric. Sci., 44, 193-198.

Hugues G. P., Reid D., I95r. Studies on the behaviour of cattle and sheep in relation to the utilization of grass. J. agric. Sci., 41, 350-366.

Hull J. L., Lofgreen G. P., Meyer J. H., r96o. Continious versus intermittent observations in behaviour studies with grazing cattle. J. Anim. Sci., 19, 1204-1207.

Johnstone-Wallace D. B., KEnNEDY K., I944. Grazing management practices and their relationship in the behaviour and grazing habits of cattle. J. Agric. Sci., 34, I 9o-r 97.

Nelson A. B., FurR R. D., I966. Interval of observation of grazing habits of range beef cows. $J$. Range $M$ gt., 19, I6-29.

Sheppard A. J., BlASER R. E., Kincaid C. M., I957. The grazing habits of beef cattle on pasture. J. Anim. Sci., 16, 68I-687.

WAGNon K. A., I963. Behaviour of beef cows on a california range. California Agric. Exp. Sta. Bull., $799,58 \mathrm{p}$.

WALKer D. E., I962. Suckling and grazing behaviour of beef heifers and calves. N. Z. J. Agric. Res., 5, 33 I- 338 . 